\title{
miR-216a rescues dexamethasone suppression of osteogenesis, promotes osteoblast differentiation and enhances bone formation, by regulating c-Cbl-mediated PI3K/AKT pathway
}

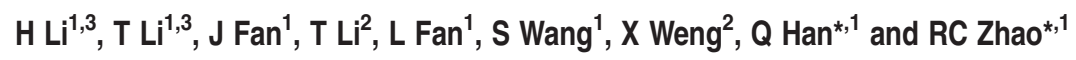

Osteoporosis is a disease marked by reduced bone mass, leading to an increased risk of fractures or broken bones. Bone formation is mediated by recruiting mesenchymal stem cells (MSCs). Elucidation of the molecular mechanisms that regulate MSC differentiation into osteoblasts is of great importance for the development of anabolic therapies for osteoporosis and other bone metabolism-related diseases. microRNAs (miRNAs) have been reported to have crucial roles in bone development, osteogenic differentiation and osteoporosis pathophysiology. However, to date, only a few miRNAs have been reported to enhance osteogenesis and regulate the suppressive effect of glucocorticoids on osteogenic differentiation. In this study, we discovered that miR-216a, a pancreatic-specific miRNA, was significantly upregulated during osteogenic differentiation in human adiposederived MSCs (hAMSCs). The expression of miR-216a was positively correlated with the expression of bone formation marker genes in clinical osteoporosis samples. Functional analysis demonstrated that miR-216a can markedly promote osteogenic differentiation of hAMSCs, rescue the suppressive effect of dexamethasone (DEX) on osteogenic differentiation in vitro and enhance bone formation in vivo. c-Cbl, a gene that encodes a RING finger E3 ubiquitin ligase, was identified as a direct target of miR-216a. Downregulation of $c-C b l$ by short hairpin RNAs can mimic the promotion effects of miR-216a and significantly rescue the suppressive effects of DEX on osteogenesis. Pathway analysis indicated that miR-216a regulation of osteogenic differentiation occurs via the c-Cbl-mediated phosphatidylinositol 3 kinase (PI3K)/AKT pathway. The recovery effects of miR-216a on the inhibition of osteogenesis by DEX were attenuated after blocking the PI3K pathway. Thus, our findings suggest that miR-216a may serve as a novel therapeutic agent for the prevention and treatment of osteoporosis and other bone metabolism-related diseases. Cell Death and Differentiation (2015) 22, 1935-1945; doi:10.1038/cdd.2015.99; published online 24 July 2015

Osteoporosis is a systemic skeletal disease characterized by low bone mass and micro-architectural deterioration of bone tissue, with a consequent increase in bone fragility. ${ }^{1}$ With the advent of a large aging population, as well as the wide clinical use of glucocorticoids, osteoporosis has become the 'invisible killer' in a large part of the population. Senile osteoporosis and postmenopausal osteoporosis are the main types of primary osteoporosis. Glucocorticoids are frequently used to treat inflammatory and autoimmune disorders. However, long-term administration of glucocorticoids has several adverse effects, of which osteoporosis is one with a high incidence and a wide range of damaging effects. Glucocorticoids are the leading cause of secondary osteoporosis. Glucocorticoids suppress bone formation and osteoblastgenesis through their direct and indirect effects on osteoblasts, osteoclasts and osteocytes, increasing bone resorption and eventually leading to a negative bone balance. ${ }^{2,3}$ So far, there are no effective preventive measures for glucocorticoid-induced osteoporosis (GIO).

Bone formation is mediated by recruiting mesenchymal stem cells (MSCs), which then differentiate into osteoblasts. MSCs, which are present in the stroma of virtually all mammalian organs, particularly in the bone marrow and subcutaneous fat, ${ }^{4-7}$ can differentiate into osteoblasts, adipocytes and chondrocytes. ${ }^{8,9}$ It has been previously reported that the osteoblast number declines remarkably during ageing or osteoporosis, the loss of which is accompanied by a significant decrease in transcripts for mesenchymal cells and osteoprogenitor markers, as well as a reduction in bone mass. ${ }^{10,11}$ Thus, the identification of factors that regulate the osteogenic differentiation of MSCs is an area of intensive investigation with the potential for identifying novel targets to enhance bone formation for osteoporosis.

\footnotetext{
${ }^{1}$ Department of Cell Biology, Institute of Basic Medical Sciences Chinese Academy of Medical Sciences, School of Basic Medicine Peking Union Medical College, Peking Union Medical College Hospital, Center of Excellence in Tissue Engineering Chinese Academy of Medical Sciences, Beijing, China and ${ }^{2}$ Department of Orthopaedic Surgery, Peking Union Medical College Hospital, Peking Union Medical College, Chinese Academy of Medical Science, Beijing, 100730, China

${ }^{*}$ Corresponding author: Q Han or RC Zhao, Institute of Basic Medical Sciences Chinese Academy of Medical Sciences, School of Basic Medicine Peking Union Medical College, Center of Excellence in Tissue Engineering Chinese Academy of Medical Sciences, Beijing, 100005, China. Tel: +86 1065123207 or +86 1065125311 ; Fax: +861065125311 or +861065125311; E-mail: hanqinhanqin@126.com or zhaochunhua@ vip.163.com

${ }^{3}$ These authors contributed equally to this work.

Abbreviations: MSCs, mesenchymal stem cells; miRNAs, microRNAs; hAMSCs, human adipose-derived MSCs; hucMSCs, human umbilical cord-derived MSCs; DEX, dexamethasone; GIO, glucocorticoid-induced osteoporosis; 3UTR, 3'-untranslated region; ALP, alkaline phosphatase; RUNX2, runt-related transcription factor; OSX, osterix; OPN, osteopontin; qRT-PCR, quantitative real-time PCR; HA/TCP, hydroxyapatite/tricalcium phosphate; shRNA, short hairpin RNA

Received 04.1.15; revised 19.6.15; accepted 22.6.15; Edited by P Salomoni; published online 24.7.15
} 
microRNAs (miRNAs) are highly conserved, small ( 22 nucleotides long), single-stranded endogenous noncoding RNAs that have been identified as regulators of diverse biological processes, such as cell proliferation, cell cycle, differentiation, organ development, cancer and hormone secretion. ${ }^{12-16}$ miRNAs typically regulate gene expression at the post-transcriptional level by promoting mRNA degradation and/or inhibiting mRNA translation through binding to complementary sequences in the 3'-untranslated region (3'UTR) of the target mRNAs. ${ }^{17,18}$ It has been reported that $\sim 30 \%$ of human protein-coding genes are regulated by miRNAs, indicating the essential role of miRNAs in regulating gene expression. ${ }^{19,20}$ A growing body of evidence has suggested that miRNAs have crucial roles in different aspects of bone development, osteogenic differentiation and osteoporosis pathophysiology. ${ }^{21}$ Several miRNAs (e.g., miR-133, miR-135, miR-138, miR-34s, miR-1192, miR-214, miR-34a and miR$-542 a-3 p)$ have been reported to be negative regulators of osteogenic differentiation and bone formation through their targeting of the osteogenic master transcription factors RUNX2 (runt-related transcription factor), osterix (OSX) or other osteogenic-related genes. ${ }^{22-29}$ In addition, a few miRNAs have been identified to function as 'molecular switches' responsible for the balance of MSC differentiation to osteoblasts versus adipocytes. For example, miR-204, miR-17-5p and miR-106a suppress osteoblast differentiation and promote adipocyte differentiation, whereas miR-637 has the opposite effects. ${ }^{30-32}$ However, to date, only a few miRNAs have been reported to be antagonists for the suppression of glucocorticoids during osteogenic differentiation. ${ }^{33,34}$

In this study, we revealed that miR-216a promotes the osteogenic differentiation of human adipose-derived MSCs (hAMSCs) in vitro and enhances bone formation in vivo. Moreover, we demonstrated that a high concentration of dexamethasone (DEX) inhibits osteogenic differentiation, whereas miR-216a antagonizes the suppressive effect of DEX on osteogenic differentiation. $\mathrm{c}-\mathrm{Cbl}$, a gene encoding a RING finger E3 ubiquitin ligase, was identified as a direct target of miR-216a. Pathway analysis indicates that the promotion of miR-216a in osteogenic differentiation occurs by regulating the $\mathrm{c}-\mathrm{Cbl}$-mediated phosphatidylinositol 3 kinase (PI3K)/AKT pathway. Importantly, downregulation of c-Cbl can also significantly attenuate the suppressive effect of DEX on osteogenic differentiation of hAMSCs. The recovery effects of miR-216a on the inhibition of osteogenic differentiation by DEX are attenuated by blocking the PI3K pathway. Our findings suggest that miR-216a may serve as a novel therapeutic agent for the prevention and treatment of osteoporosis and other bone metabolism-related diseases.

\section{Results}

Expression pattern of miR-216a. miRNA arrays were conducted to identify miRNAs that are associated with the osteogenic differentiation of hAMSCs. Expression pattern of miRNAs were compared between osteogenic-induced 4-day cells and undifferentiated-hAMSCs. According to fold change and $P$-values, we selected 12 significantly upregulated miRNAs to investigate the function (Figure $1 \mathrm{a}$ and
Supplementary Table S1). We found some of these miRNAs can promote osteogenic differentiation, among which the promoting effect of miR-216a was most significant, thus we chose miR-216a in follow-up experiments. TaqMan quantitative real-time PCR (qRT-PCR) further confirmed that miR-216a sharply increased when hAMSCs underwent osteogenic differentiation (Figure 1b). Sequence analysis of the miR-216a revealed that it is highly conserved in Homo sapiens, Mus musculus, Rhesus macaque, Danio rerio, Pan troglodytes, Pongo pygmaeus and Capitella teleta (Figure 1c). Based on further qRT-PCR analysis of various mouse tissues, miR-216a exhibited low expression in several tissues (e.g., lung, liver, heart, kidney and colon), whereas it was highly expressed in pancreas, osteogenic progenitors and osteoblasts (Figure 1d). Similarly, miR-216a expression in several ordinary cell lines was also much lower than that in osteoblasts (Figure 1e).

miR-216a regulates osteogenic differentiation in vitro and bone formation in vivo. To investigate the roles of miR-216a in the regulation of osteogenic differentiation, we inhibited the endogenous expression of miR-216a in hAMSCs by antagomir (Figure 2a) and then induced miR-216a antagomir (anta-216a)-transfected or negative control antagomir (anta-NC)-transfected hAMSCs to differentiate into the osteogenic lineage. qRT-PCR and western blot analyses showed that mRNA and protein levels of the osteogenic transcription factors and marker genes alkaline phosphatase (ALP), osteopontin (OPN), RUNX2, COL1A1 and IBSP were significantly decreased in anta-216atransfected hAMSCs compared with anta-NC-transfected cells during osteogenic differentiation (Figures $2 b$ and $c$ ). Repressed ALP staining and ALP activity (Figures 2d and e), as well as decreased mineral deposition detected by Alizarin red staining (Figure 2f), further indicated that inhibition of miR-216a significantly suppressed the osteogenic differentiation of hAMSCs.

We next infected hAMSCs with lenti-miR-216a (lenti-216a) or lenti-miR-negative control (lenti-NC) and purified them with puromycin. The intracellular miR-216a levels were remarkably upregulated following lenti-216a infection (Figure 3a). The opposite effects were observed, as indicated by a significant elevation of osteogenic transcription factors and marker gene expression (Figures $3 b$ and $c$ ), enhanced ALP staining and ALP activity (Figures $3 d$ and e) and increased in vitro mineralization in lenti-216a-infected hAMSCs compared with control lenti-NC-infected cells during osteogenic differentiation (Figure 3f). A similar role for miR-216a in the positive regulation of osteogenic differentiation was also observed in human umbilical cord-derived MSCs (hucMSCs) (Supplementary Figures S1A-E).

To investigate the role of miR-216a in vivo, a preclinical ectopic bone formation model in NOD/SCID mice was used. Uninfected hAMSCs and lenti-216a- or lenti-NC-infected hAMSCs were loaded onto hydroxyapatite/tricalcium phosphate (HA/TCP) scaffolds, respectively, and implanted subcutaneously into NOD/SCID mice for 8 weeks. Hematoxylin and eosin (H\&E) staining revealed a remarkable increase in the quantity and size of osteoids in the mouse grafts with lenti-216a-infected hAMSCs, whereas no obvious osteoids 


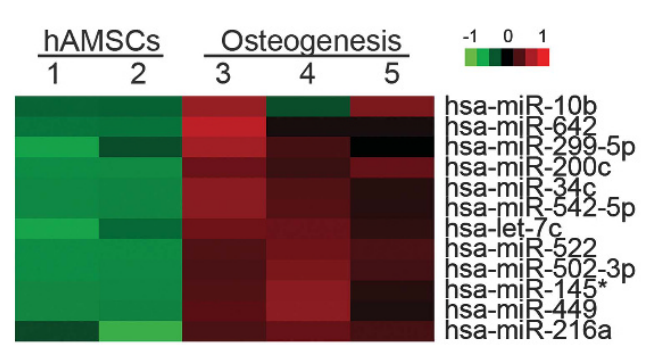

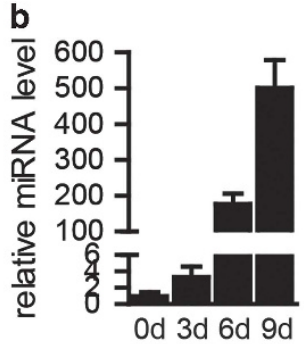

Od 3d 6d 9d

C

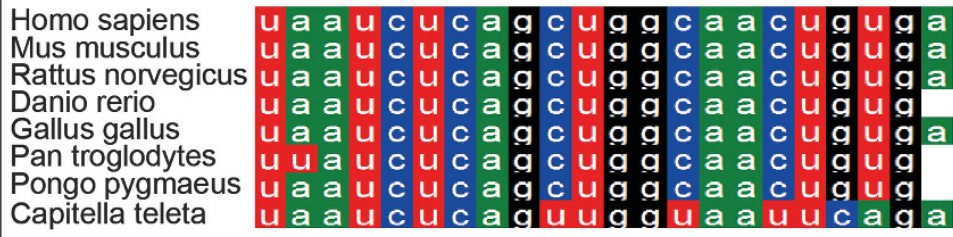
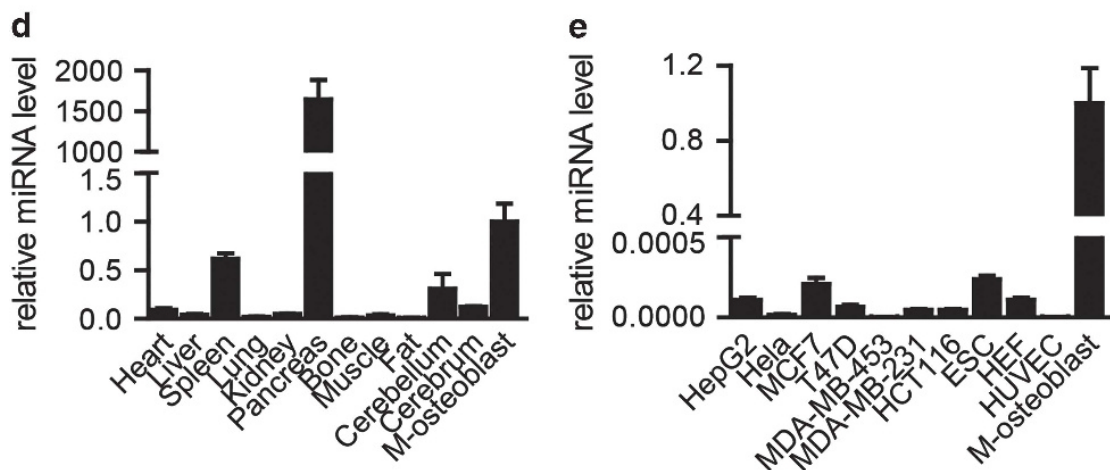

Figure 1 Expression pattern of miR-216a. (a and $\mathbf{b}$ ) The dynamic expression profile of miR-216a during osteogenesis in hAMSCs was detected by miRNA microarray and validated by TaqMan qRT-PCR. (c) Analysis of miR-216a conservation in mammals. (d and e) The tissue and cell line expression patterns of miR-216a compared with MSCderived osteoblasts (M-osteoblast) were analyzed by TaqMan qRT-PCR. U6 was used as an internal control in the miRNA-specific TaqMan qRT-PCR. Quantitative data are presented as the mean \pm S.D. $(n=3)$

were detected in the grafts with either lenti-NC-infected hAMSCs or uninfected control hAMSCs (Figure $3 \mathrm{~g}$ ). Therefore, we demonstrated that overexpression of miR-216a not only promotes osteogenic differentiation in vitro but also facilitates ectopic bone formation in vivo.

miR-216a antagonizes the suppressive effect of DEX on osteogenic differentiation and is correlated with bone formation. It has been reported that long-term administration of glucocorticoids can lead to $\mathrm{GIO} .^{35}$ Glucocorticoids suppress bone formation through their direct and indirect effects on osteoblasts. ${ }^{2,3}$ DEX, an extensively used synthetic glucocorticoid, has been found to suppress osteogenic differentiation. ${ }^{36}$ To clarify the effect of glucocorticoids on osteogenic differentiation, we used different concentrations of DEX to treat hAMSCs in the process of osteogenic differentiation. Using ALP staining, Alizarin red staining and qRT-PCR, we demonstrated that DEX suppressed the osteogenic differentiation of hAMSCs in a dose-dependent manner. When the DEX concentration was higher than $10 \mathrm{nM}$, it significantly inhibited osteoblast differentiation, as demonstrated by decreased ALP staining, reduced matrix mineralization, and suppressed expression of osteogenic factors and marker genes (Supplementary Figures S2A-C).
To investigate whether miR-216a overexpression can affect the inhibition of osteoblast differentiation caused by DEX in hAMSCs, we induced lenti-216a-infected hAMSCs into osteogenic lineage with osteogenesis induction medium containing $10 \mathrm{nM}$ DEX. We found that the ALP staining and ALP activity were significantly enhanced by miR-216a overexpression (Figures $4 a$ and $b$ ). Alizarin red staining also showed that in vitro matrix mineralization increased in DEXtreated cells after miR-216a overexpression (Figure 4c). In addition, the expression of the osteoblast-specific transcription factors and marker genes RUNX2, ALP, OPN, COL1A1 and IBSP were mostly rescued at mRNA and protein levels in lenti-216a-infected hAMSCs compared with lenti-NC-infected cells under DEX treatment (Figures $4 d$ and e). These results confirmed that miR-216a overexpression significantly rescued the inhibition effects of DEX on osteoblast differentiation.

Our findings provide new opportunities for the prevention and treatment of osteoporosis and other bone metabolismrelated diseases. We collected 67 clinical samples from osteoporosis patients of different ages and analyzed the correlation between the expression of miR-216a and that of the bone formation marker genes RUNX2, ALP and OPN (Supplementary Table S1). Results showed that the expression levels of these three bone formation marker 
a

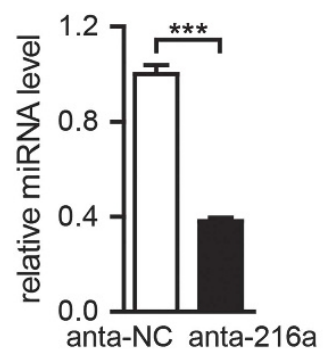

C

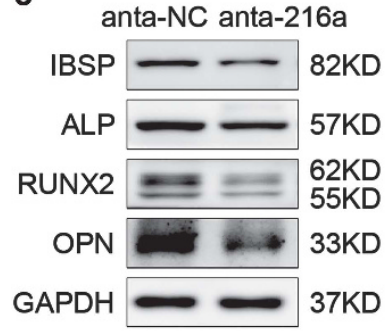

b
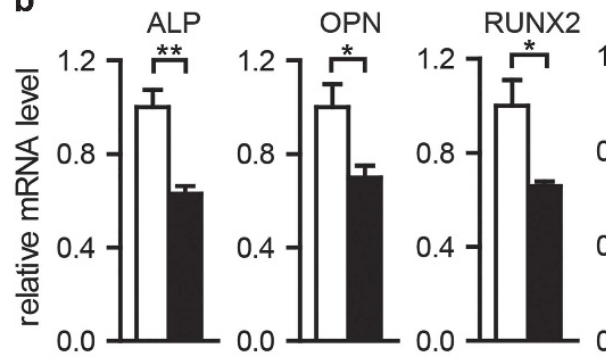

COL1A1

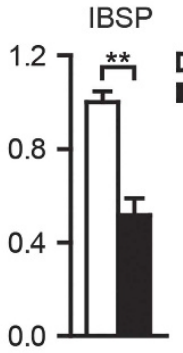

d

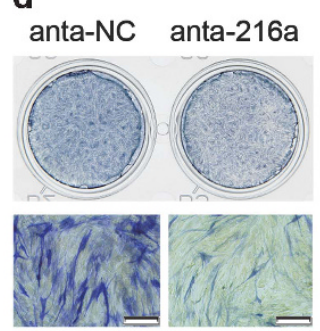

e

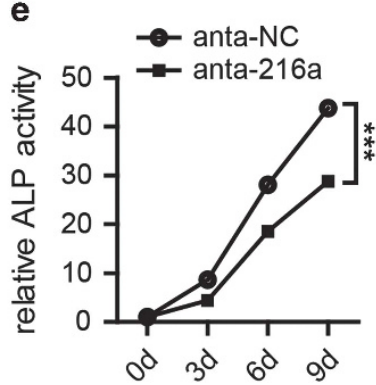

f anta-NC anta-216a

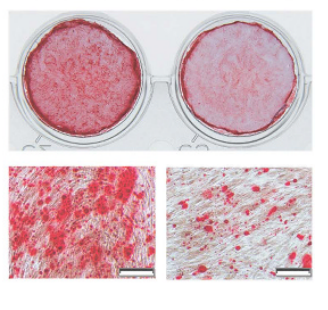

Figure 2 Downregulation of miR-216a inhibits the osteogenic differentiation of hAMSCs. (a) miR-216a expression was determined by TaqMan qRT-PCR in miR-216a antagomir (anta-216a)-transfected hAMSCs after $48 \mathrm{~h}$ compared with that in negative control antagomir (anta-NC)-transfected cells. (b and $\mathbf{c}$ ) qRT-PCR and western blot analyses of the expression of osteoblast marker genes in anta-216a-transfected and anta-NC-transfected cells on day 6 of osteogenic differentiation. (d) ALP staining was performed on day 6 of osteogenic differentiation. (e) ALP activity was measured during osteogenic differentiation. (f) Alizarin red staining was performed to indicate mineral deposition on day 12. Scale bars: $200 \mu \mathrm{m}$. GAPDH was used as an internal control in the qRT-PCR and western blot analyses. U6 was used as an internal control in the miRNAspecific TaqMan qRT-PCR. Quantitative data are presented as the mean \pm S.D. $(n=3)$. ${ }^{\star} P<0.05 ;{ }^{* \star} P<0.01 ;{ }^{* \star} P<0.001$ compared with the control

genes were positively correlated with each other (Supplementary Figure S3). Furthermore, the expression of miR-216a was positively correlated with the expression of the bone formation marker genes RUNX2, ALP and OPN (Figure 4f). To preliminarily assess the safety of miR-216 for clinical use, we determined the karyotype of miR-216aoverexpressed hAMSCs and their derived osteoblasts. The data showed normal karyotype in all of the miR-216aoverexpressed hAMSCs and their derived cells (Supplementary Figures S4A-C). We also analyzed the proliferation and cell cycle of miR-216a-overexpressed hAMSCs by MTS and flow cytometry, respectively. We observed that miR-216a overexpression did not significantly affect the proliferation and cell cycle of hAMSCs (Supplementary Figures S5A-C).

miR-216a directly targets $\mathbf{c}$-Cbl. To gain insight into the molecular mechanisms by which miR-216a regulates the osteogenic differentiation of hMSCs, we predicted the potential targets of miR-216a using TargetScan and found that osteogenic-related genes c-Cbl, Smad7, NLK, CEBPG and TGFBR2 have miR-216a binding sites in their 3UTR (Figure 5a and Supplementary Figure S6A). To test whether miR-216a directly targets these genes, we constructed luciferase reporters that had either a wild-type (WT) 3UTR or a $3^{\prime} U T R$ containing mutant sequences of the miR-216a binding site. We found that overexpression of miR-216a remarkably inhibited the luciferase reporter activity of the WT c-Cbl 3'UTR and WT Smad7 3'UTR, but not that of the mutated 3'UTR or another gene's 3'UTR (Figures 5b and c and Supplementary Figure S6B). Moreover, the expression of
c-Cbl in hAMSCs was markedly downregulated at protein level, but not at mRNA level, after miR-216a overexpression, whereas mRNA and protein levels of Smad7 exhibited no significant difference between the lenti-miR-216a- and lenti$\mathrm{NC}$-infected groups (Figures $5 \mathrm{~d}$ and $\mathrm{e}$ ). These findings suggest that miR-216a regulates $\mathrm{c}-\mathrm{Cbl}$ expression at the post-transcriptional level in hAMSCs.

To determine whether miR-216a functionally targets c-Cbl in regulating hAMSCs osteogenic differentiation, we used two short hairpin RNAs (shRNAs) to knock down the endogenous expression of c-Cbl. The qRT-PCR and western blot analyses indicated that both shRNAs effectively suppressed mRNA and protein expression of $\mathrm{c}$-Cbl (Supplementary Figure S7A). However, Cbl-b, another Cbl family member, exhibited no significant changes at mRNA level by these two shRNAs (Supplementary Figure S7A), suggesting the c-Cbl-specific suppression by these shRNAs. Next, using the c-Cbl shRNAs, we found that downregulation of endogenous c-Cbl remarkably promoted osteogenic differentiation in hAMSCs, as demonstrated by enhancement of ALP staining and ALP activity, promotion of matrix mineralization in vitro (Figures $5 f-h$ ) and significant upregulation of the expression of the osteogenic regulators and osteoblast marker genes (RUNX2, ALP, OPN, IBSP and COL1A1) at mRNA and protein levels (Figures $5 \mathrm{i}$ and $\mathrm{j}$ ). Moreover, when endogenous $\mathrm{c}-\mathrm{Cbl}$ was downregulated by specific shRNAs (sh-c-Cbl) in hAMSCs during osteogenic differentiation with DEX treatment, the suppressive effect of DEX on osteoblast differentiation was also significantly rescued (Supplementary Figures S7B-D). The above results were consistent with those observed in miR-216a-overexpressed hAMSCs. 
a

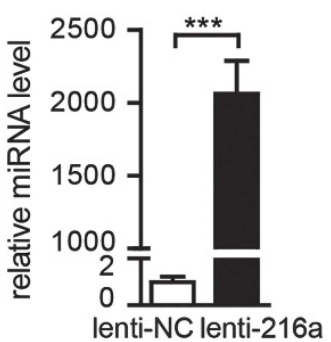

C

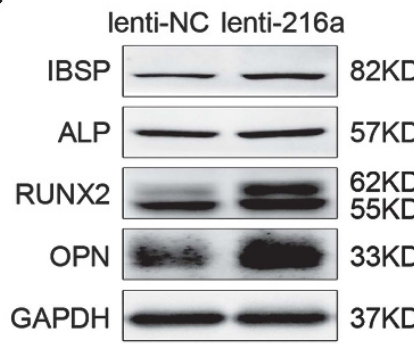

b

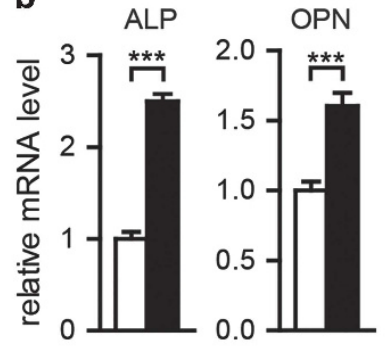

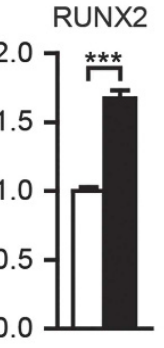
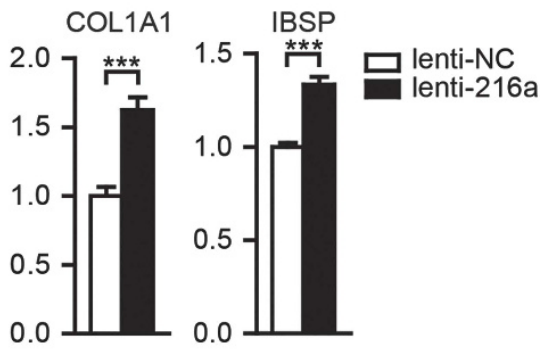

lenti-NC lenti-216a
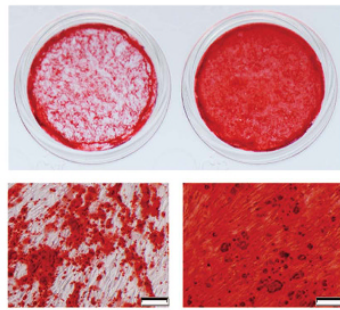

d

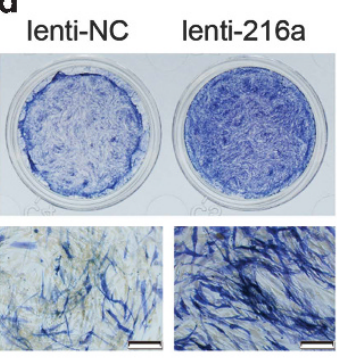

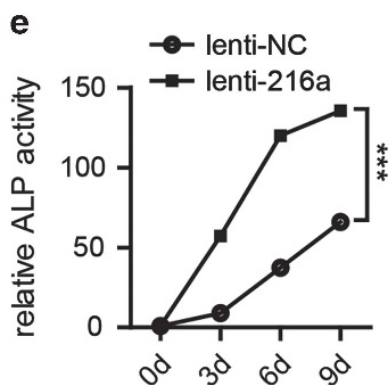

g
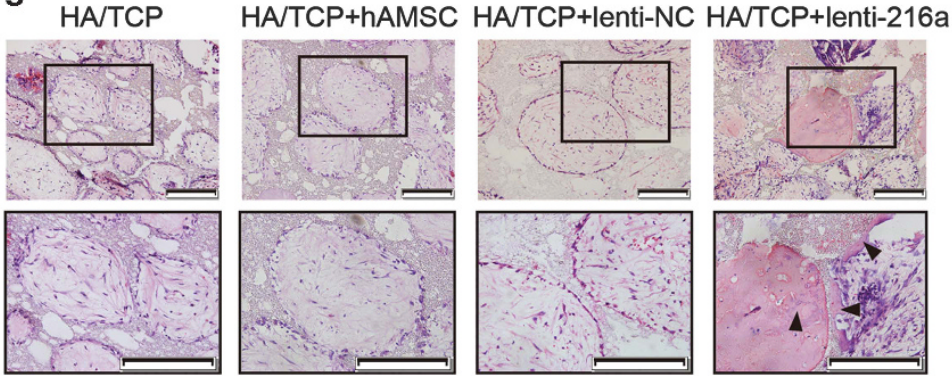

Figure 3 miR-216a promotes the osteogenic differentiation and bone formation of hAMSCs. (a) miR-216a expression was determined by TaqMan qRT-PCR in lenti-216ainfected hAMSCs compared with that in lenti-NC-infected cells. ( $\mathbf{b}$ and $\mathbf{c}$ ) qRT-PCR and western blot analyses of the expression of osteoblast marker genes in lenti-216a- and lenti-NC-infected cells on day 6 of osteogenic differentiation. (d) ALP staining was performed on day 6 of osteogenic differentiation. (e) ALP activity was measured during osteogenic differentiation. (f) Alizarin red staining was performed to indicate mineral deposition on day 12. (g) hAMSCs were infected with lenti-216a or lenti-NC and implanted into NOD/SCID mice. H\&E staining was performed after 8 weeks of implantation. Arrowheads: bone matrix. Scale bars: $200 \mu$ m. GAPDH was used as an internal control in the qRT-PCR and western blot analyses. U6 was used as an internal control in the miRNA-specific TaqMan qRT-PCR. Quantitative data are presented as the mean \pm S.D. $(n=3)$. ${ }^{* *} P<0.001$ compared with the control

miR-216a regulates the c-Cbl-mediated PI3K/AKT pathway during osteogenic differentiation in hAMSCs. $c-C b l$ is an adaptor protein and E3 ligase that has important roles in several signaling pathways that affect various cellular functions. ${ }^{37,38}$ Among them, the PI3K and STAT5 pathways have been reported to be involved in the regulation of bone formation and osteoblast differentiation by interacting with c-Cbl. ${ }^{39-41} \mathrm{PI} 3 \mathrm{~K}$ inhibitor LY294002 or STAT5 inhibitor SC-355979 was added into the osteogenesis induction medium during osteogenic differentiation of hAMSCs. We demonstrated that PI3K inhibitor LY294002 remarkably suppressed the osteogenic differentiation of hAMSCs in a concentration-dependent manner, which was confirmed by the reduction of ALP staining and ALP activity and downregulation of the PI3K/AKT signaling pathway-related molecular elements and osteogenic factors (Figures $6 a-c$ ). However, STAT5 inhibitor SC-355979 showed no significant effect on this process (data not shown). Therefore, we focused on the PI3K pathway in subsequent experiments. Western blot showed that overexpression of miR-216a increased the expression levels of p85 and phospho-Akt, indicating that the PI3K/AKT signal pathway was enhanced (Figure 6d). This finding was consistent with the effect of knockdown of $\mathrm{c}-\mathrm{Cbl}$ with shRNAs on protein levels of p85 and phospho-Akt (Figure 6e). In addition, the enhancement of hAMSC osteogenic differentiation by miR-216a overexpression was almost blocked by LY294002, as demonstrated by decreased ALP expression and ALP staining and ALP activity and reduced mineral deposition (Figures 6f-i). Furthermore, the recovery effects of miR-216a on the inhibition of osteogenesis by DEX were also attenuated by the PI3K inhibitor LY294002 (Figures 7a-d). Together, these results suggest that miR-216a regulation of osteogenic differentiation and bone formation of hAMSCs occurs via the c-Cbl/ PI3K signaling pathway (Figure 8).

\section{Discussion}

More and more miRNAs are found as negative factors involved in the regulation of osteogenic differentiation. ${ }^{23,26,27}$ Only a 
a

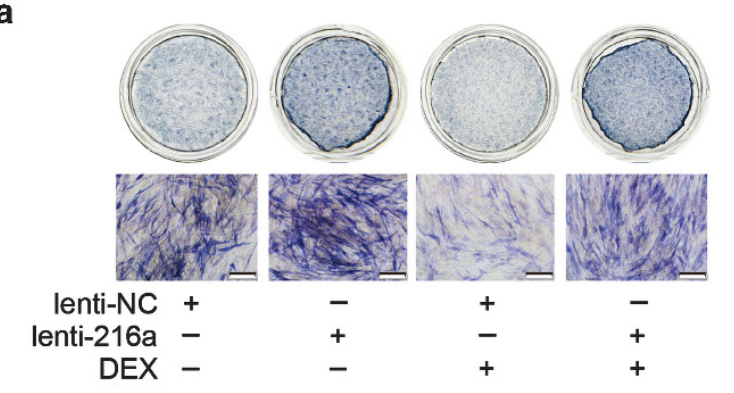

c
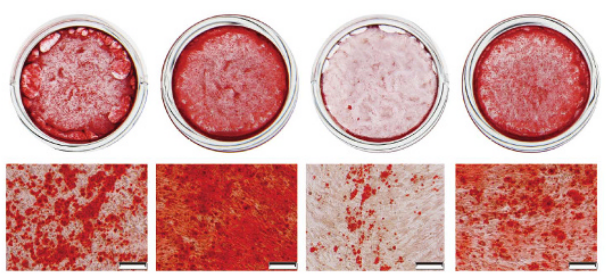

lenti-NC + lenti-216a -

DEX -

\begin{abstract}
$+$
\end{abstract}
$-$
$+$

$+$

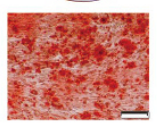

$-$
$+$
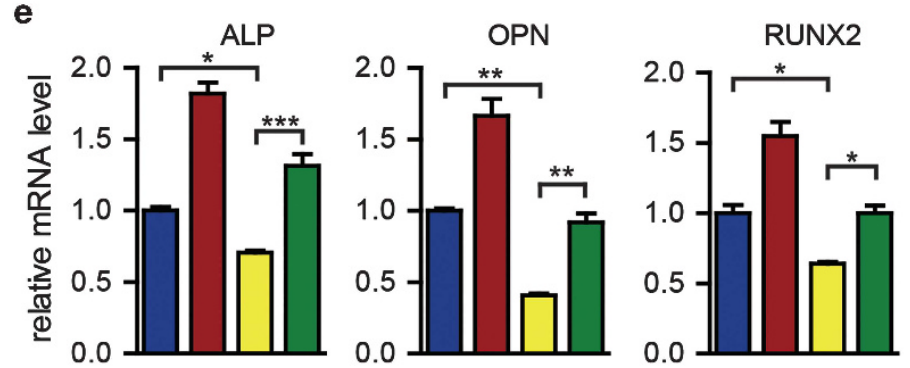

b

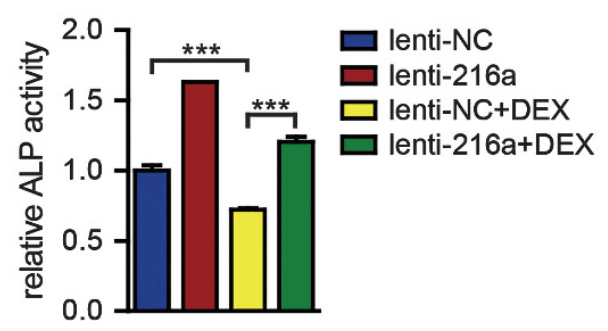

d
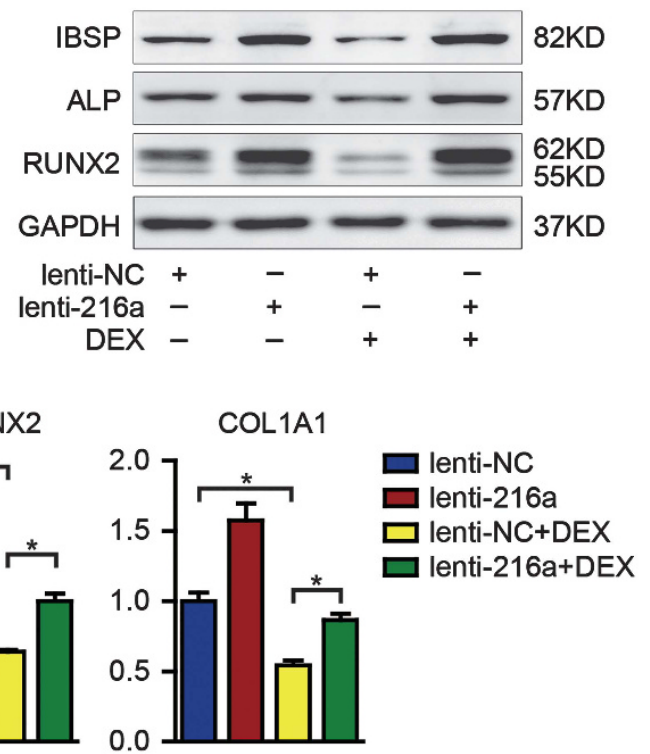

f
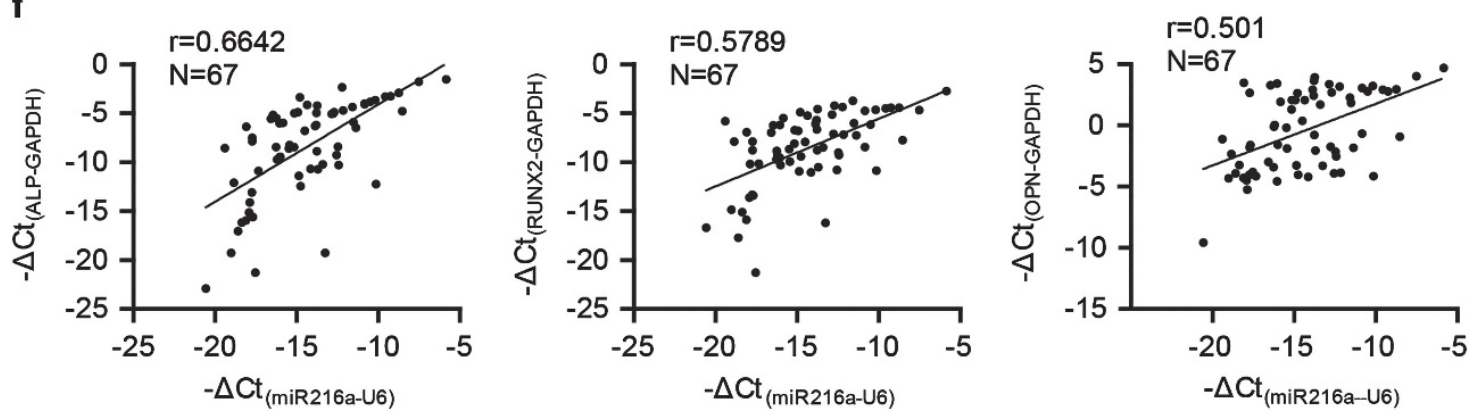

Figure 4 miR-216a rescues the effect of DEX on osteogenic differentiation and is correlated with bone formation. (a and $\mathbf{b})$ ALP staining and ALP activity detection were performed on day 6 of osteogenic differentiation. (c) Mineral deposition was indicated by Alizarin red staining on day 12. (d and e) Osteogenic differentiation was confirmed by qRT-PCR and western blot analyses of the osteogenic transcription factors and marker genes on day 6 . (f) The correlation between the expression of bone formation makers and expression of miR-216a was analyzed in 67 osteoporosis samples. DEX: $10 \mathrm{nM}$ DEX. Scale bars: $200 \mu \mathrm{m}$. GAPDH was used as an internal control in the qRT-PCR and western blot analyses. Quantitative data are presented as the mean \pm S.D. $(n=3) .{ }^{*} P<0.05 ;{ }^{* *} P<0.01 ;{ }^{* \star *} P<0.001$ compared with the control

few miRNAs have been found to promote osteogenic differentiation. ${ }^{25,42,43}$ In this study, we first identified miR-216a as an important enhancer of osteoblast differentiation of hAMSCs and hucMSCs. In the case of bone formation, MSCs generate new osteoblasts and their progenitors, which are essential for bone homeostasis and fracture healing. ${ }^{44,45}$ Using a preclinical ectopic bone formation model, we found that miR-216a can effectively promote bone tissue generation in vivo, which suggested that miR-216a may be an effective agent for promoting bone tissue engineering, bone defect repair and fracture healing.

DEX, a widely used glucocorticoid, was shown to inhibit osteogenic differentiation and bone formation, ${ }^{36,46}$ and DEX redirects the differentiation of bone marrow stromal cells from the osteoblastic lineage to the adipocyte lineage. ${ }^{36,47} \mathrm{We}$ found that when the concentration is higher than $10 \mathrm{nM}$, the osteogenic differentiation of hAMSCs can be significantly inhibited by DEX. In all, $10 \mathrm{nM}$ of DEX was then used as the 
a

a

c-Cbl 3'UTR WT

hsa-miR-216a

c-Cbl 3'UTR MUT

Smad7 3'UTR WT

hsa-miR-216a

Smad7 3'UTR MUT

b

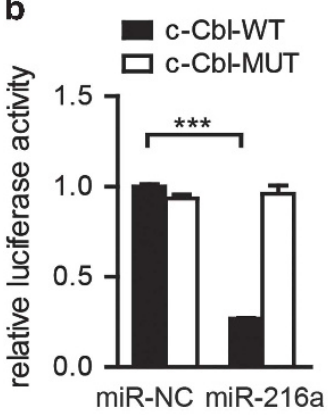

f

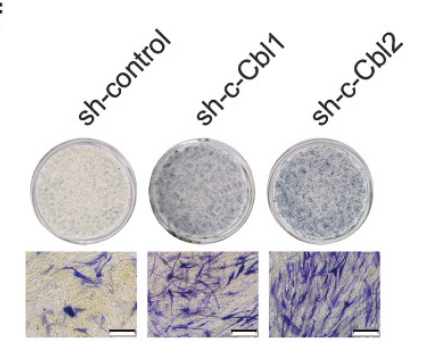

5' ...AAACAAACACAUAGGUGAGAUUU...
3' AGUGUCAACGGUCGACUCUAAU
5' ...AAACAAACACAUAGGACACUAAU...

5' ...UCCUGAAAAAAAACCUGAGAUUU. | | | | | | |

$3^{\prime}$

AGUGUCAACGGUCGACUCUAAU
...CCUGAAAAAAAACCACACUAAU...

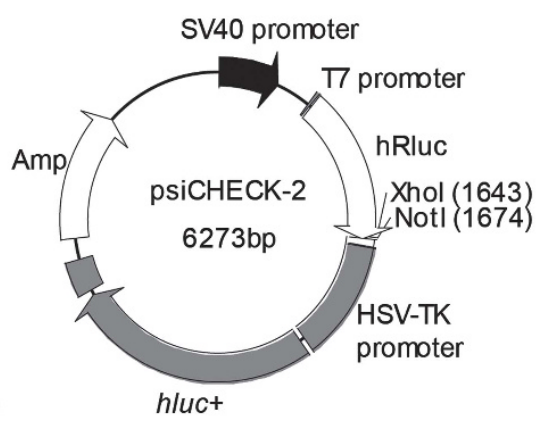

d

C Smad7-WT

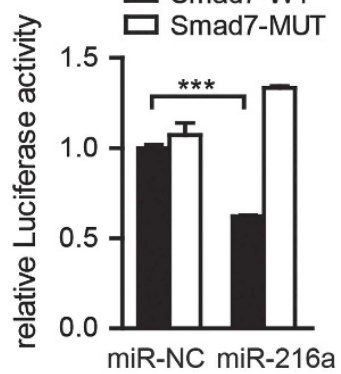

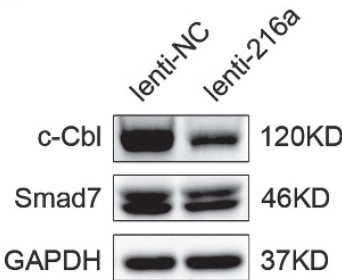

e

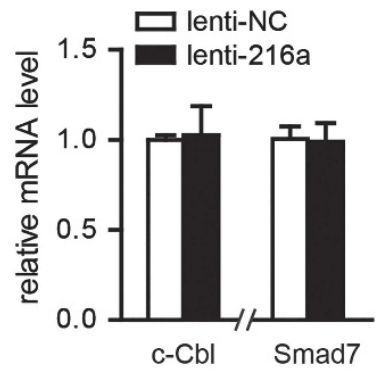

g

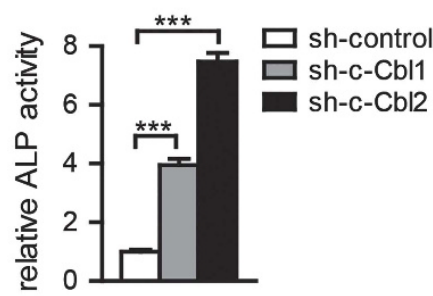

h

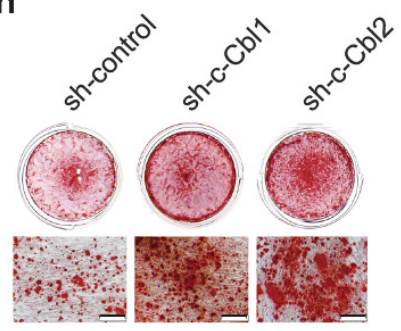

i

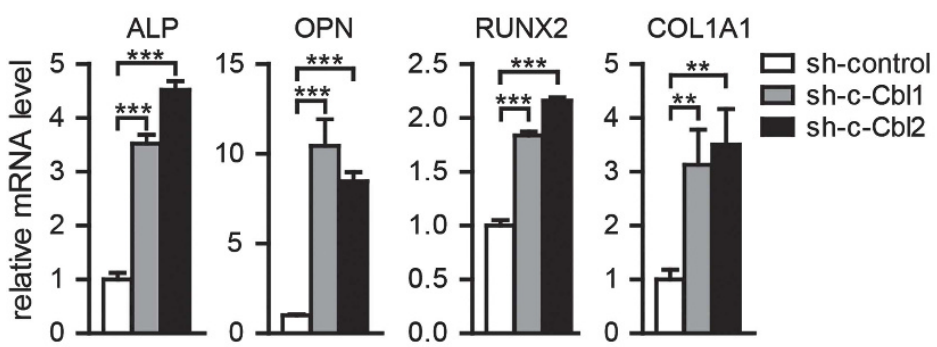

j

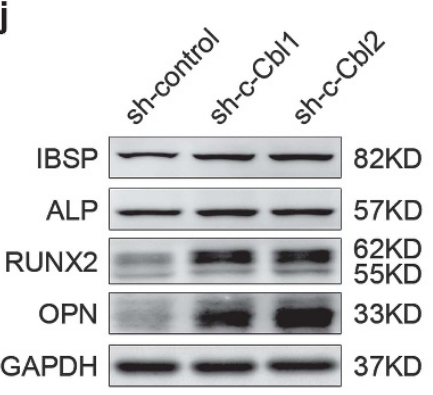

Figure 5 c-Cbl is a direct target of miR-216a. (a) Computational analysis was performed for the complementarities of the miR-216a seed sequence to the 3'UTR of c-Cbl and Smad7. The WT or mutant-type (MUT) construct was inserted into the psiCHECK-2 reporter vector. (b and $\mathbf{c}$ ) Luciferase activities were measured in the lysates, and the values were normalized to the psiCHECK vector and presented as the fold change of miR-NC. miR-216a: miR-216a mimics. (d) Western blot analysis of c-Cbl and Smad7 in hAMSCs after miR-216a overexpression. (e) qRT-PCR analyzed the expression of c-Cbl and Smad7 in hAMSCs after lenti-216a infection. (f and $\mathbf{g}$ ) ALP staining and ALP activity detection were performed on day 6 of osteogenic differentiation in sh-c-Cbl1, sh-c-Cbl2 or sh-control-infected hAMSCs. (h) Alizarin red staining was performed to indicate mineral deposition on day 12. (i and j) The qRT-PCR and western blot analyses of the osteoblast transcription factors and marker genes were performed on 6 day of osteogenic differentiation. Scale bars: $200 \mu \mathrm{m}$. GAPDH was used as an internal control in the qRT-PCR and western blot analyses. All quantitative data are presented as the mean \pm S.D. $(n=3) .{ }^{* \star} P<0.01 ;{ }^{* \star *} P<0.001$ compared with the control

minimum effective concentration in vitro system to investigate protective factors for osteoporosis. Both DEX and miRNA were found to have important roles in the regulation of osteogenic differentiation. So far only miR-29a has been reported to ameliorate glucocorticoid-induced suppression of osteoblast differentiation by regulating $\mathrm{Wnt} / \beta$-catenin pathway. ${ }^{33,34}$ In this study, we found that overexpression of miR-216a can significantly rescue the suppressive effects of DEX on the osteoblast differentiation of hAMSCs, which suggested that therapeutic upregulation of miR-216a in MSCs may promote bone formation and even reverse osteoporosis. With the development of miRNA delivery system, which can 


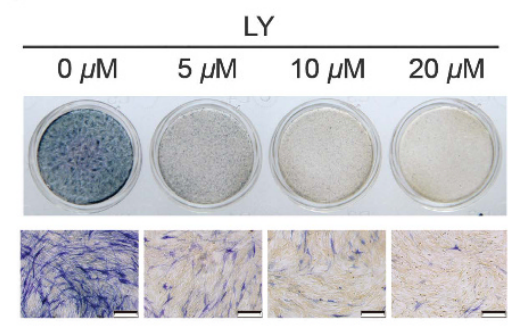

b

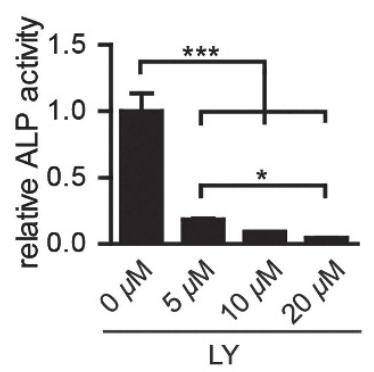

c

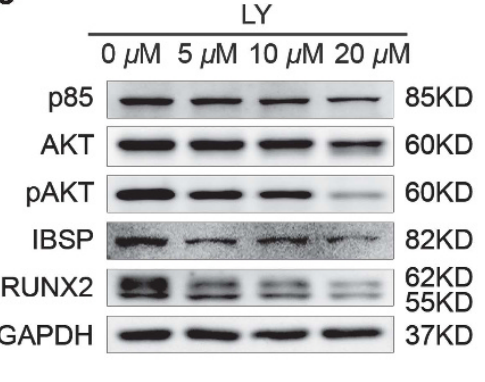

d

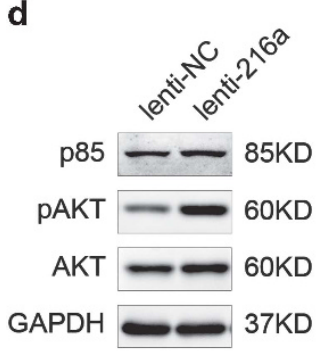

e

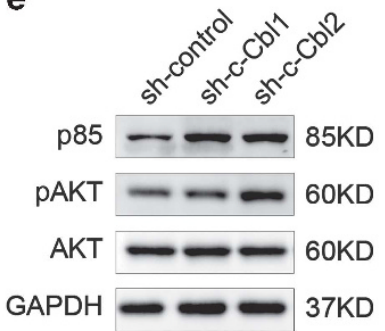

f

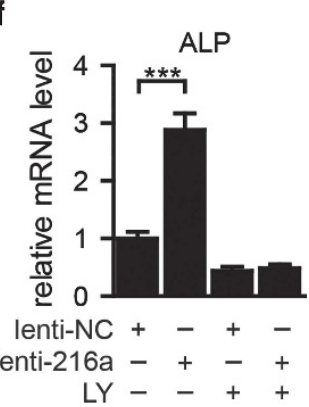

g

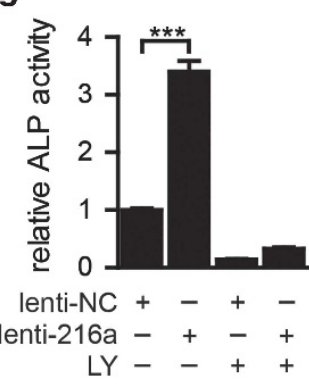

h

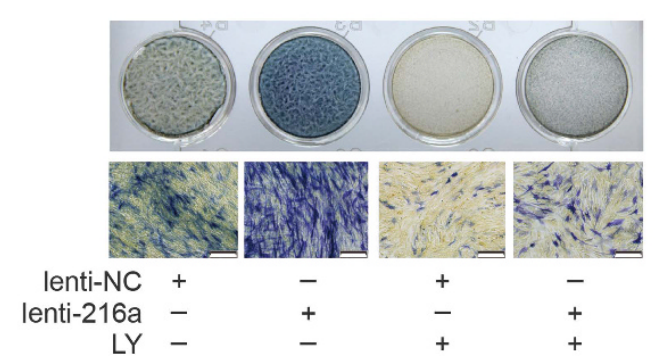

i

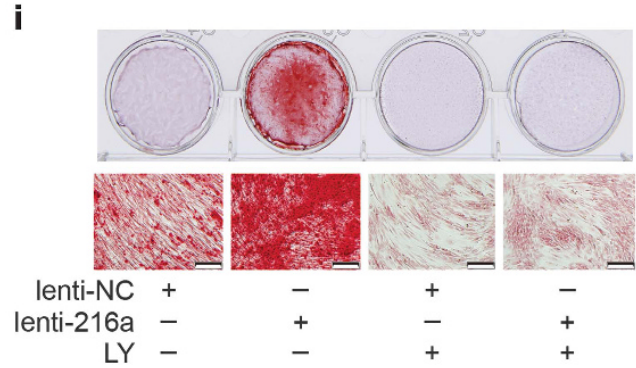

Figure 6 PI3K pathway inhibition suppresses osteogenic differentiation and attenuates the enhancement of osteogenic differentiation by miR-216a overexpression. (a and $\mathbf{b}$ ) PI3K inhibitor LY294002 was used to block the PI3K pathway. ALP staining and ALP activity detection were performed on day 6 of osteogenic differentiation in hAMSCs. (c) Western blot was performed to analyze the PI3K/AKT signaling pathway-related molecular elements and osteogenic factors after treatment with LY294002 on day 6 . (d and e) Western blot was used to detect the protein levels of p85 and phospho-Akt after the overexpression of miR-216a or downregulation of c-Cbl in hAMSCs, respectively. (f) The ALP mRNA level was analyzed by qRT-PCR in induced cells on day 6. (g and $\mathbf{h}$ ) ALP activity and ALP staining were performed on day 6 . (i) Alizarin red staining was performed to indicate mineral deposition in induced cells after different treatments on day 12. LY: PI3K inhibitor LY294002. Scale bars: $200 \mu \mathrm{m}$. GAPDH was used as an internal control in the qRT-PCR and western blot analyses. All quantitative data are presented as the mean \pm S.D. $(n=3) .{ }^{*} P<0.05 ;{ }^{* \star} P<0.001$ compared with the control

carry miRNA and specifically target bone surface, miR-216a may serve as a new therapeutic agent for osteoporosis, especially GIO.

Damage of the bone formation potential of MSCs is responsible for the bone loss incurred in aging and osteoporosis. $^{10,11}$ Recently, it was reported that elevated miR-214 levels correlated with a lower degree of bone formation in bone specimens from aged patients with osteoporosis. ${ }^{26}$ Here we were the first to demonstrate that the expression of miR-216a was positively correlated with the expression of key marker genes of bone formation, further suggesting miRNAs' great value for the prevention and treatment of osteoporosis or other bone metabolism-related diseases.

Previously, it has been reported that miR-216a is a serum biomarkers of exocrine pancreas injury of acute pancreatitis. ${ }^{48,49}$ PTEN and Smad7 were identified to be the possible targets of miR-216. ${ }^{48,49}$ In this study, we found that although miR-216a can target the 3UTR of Smad7, it has no significant effect on the protein level of this gene, which suggested that miRNAs have different targets in different cells and can act selectively in different cells. Here, we defined a new mechanism whereby miR-216a is involved in regulation the expression of $\mathrm{c}-\mathrm{Cbl}$ protein at the post-transcriptional level.

c-Cbl is a member of the RING-type E3 ubiquitin ligase family, which has important roles in several signaling pathways that affect various cellular functions. ${ }^{37,38}$ Tyrosine 731 (Tyr ${ }^{731}$ in humans; $\mathrm{Tyr}^{737}$ in mice) is unique to $\mathrm{c}-\mathrm{Cbl}$ and is phosphorylated by Src family kinases. Phosphorylated Cbl tyrosine ${ }^{731}$ provides a docking site for the p85 regulatory subunit of $\mathrm{PI} 3 \mathrm{~K}$, which has an important role in the regulation of bone homeostasis. ${ }^{39,50-52}$ It has been reported that abrogation of $\mathrm{c}-\mathrm{Cbl}-\mathrm{PI} 3 \mathrm{~K}$ interaction increase bone formation and osteoblast proliferation. ${ }^{39,40}$ We found that miR-216a functionally targets $\mathrm{c}-\mathrm{Cbl}$ to regulate the osteogenic differentiation of hAMSCs. Overexpression of miR-216a or knockdown of its target c-Cbl can increase the expression of p85 and phospho-Akt, indicating the activation of the PI3K signaling 
a
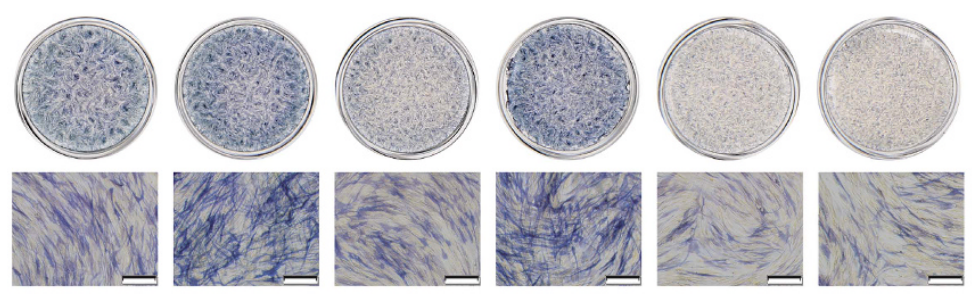

$\begin{aligned} \text { lenti-NC } & + \\ \text { Ienti-216a } & - \\ \text { DEX } & - \\ \text { LY } & -\end{aligned}$

-
+
-

$+$

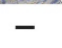

$-$

$+$

b
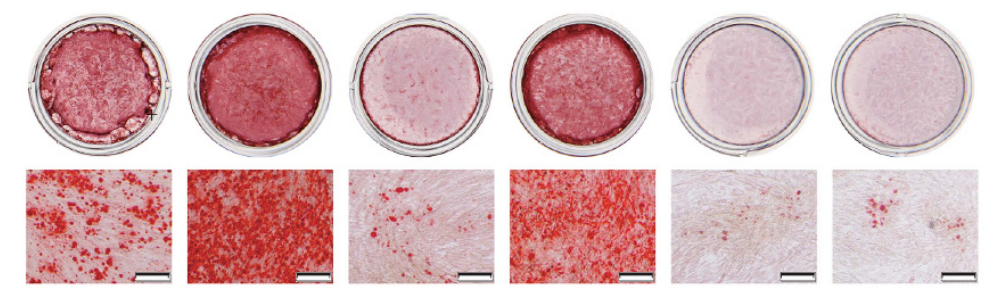

lenti-NC $\quad$
lenti-216a -
DEX -
LY -

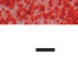

$+$

+
-

\section{$-$}

$+$

c

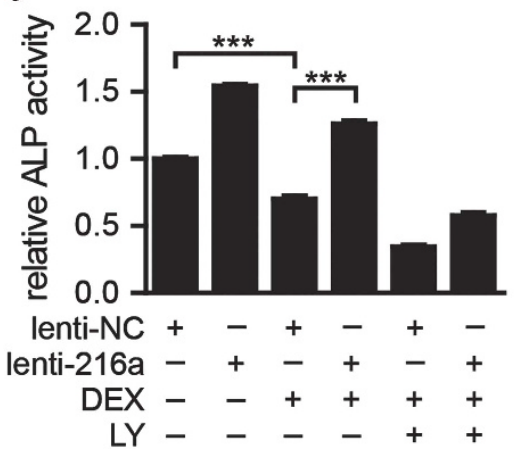

d

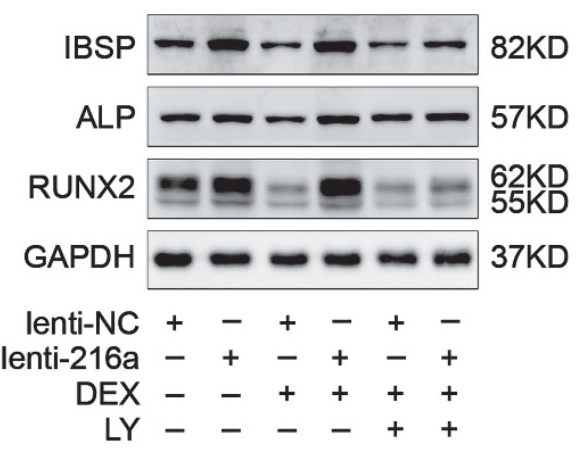

Figure 7 PI3K pathway inhibition attenuates the recovery effects of miR-216a on the inhibition of osteogenesis by DEX. (a) ALP staining was performed on day 6 after different treatments. (b) Alizarin red staining was performed to indicate mineral deposition in osteogenesis induction of hAMSCs on day 12 after different treatments. (c) The ALP activity of cells was measured on day 6 of osteogenic differentiation. (d) Western blot was performed to analyze the protein level of osteogenic transcription factors and marker genes after different treatments on day 6 of osteoblast differentiation. LY: PI3K inhibitor LY294002. Scale bars: $200 \mu \mathrm{m}$. GAPDH was used as an internal control in the western blot analysis. All of the quantitative data are presented as the mean \pm S.D. $(n=3)$. ${ }^{\star \star \star} P<0.001$ compared with the control

pathway. Both the enhancement of osteogenic differentiation and protection of DEX-induced damage during osteogenic differentiation in hAMSCs induced by miR-216a were almost completely blocked by PI3K/Akt pathway inhibitor LY294002. Those findings demonstrate that miR-216a promotes osteogenic differentiation and rescues the suppressive effects of DEX on osteogenic differentiation of hAMSCs through the c-Cbl/PI3K signaling pathway.

In summary, we are the first to confirm that miR-216a is positively correlated with bone formation and cannot only promote osteogenic differentiation in vitro and promote bone formation in vivo, but also can effectively antagonize the suppression of DEX on osteoblast differentiation, through repressing $\mathrm{c}-\mathrm{Cbl}$ expression, which, in turn, results in the activation of the PI3K signaling pathway. Notably, our findings not only enrich the understanding of miRNA regulation of bone development and osteogenic differentiation, but also enable new opportunities for the bone tissue engineering, clinical bone defect repair, and especially the prevention and treatment of osteoporosis and other bone metabolism-related diseases.

\section{Materials and Methods}

Cell isolation, culture and osteogenic differentiation. Human adipose tissues were collected from donors who performed liposuction surgery. Human umbilical cords were obtained from normal full-term deliveries. All experiments followed the procedures approved by the ethics committee at the Chinese Academy of Medical Sciences and Peking Union Medical College. hAMSCs were isolated and cultured as previously described. ${ }^{53}$ hucMSCs were generated as described previously. ${ }^{54}$

To induce osteogenic differentiation, third-passage hAMSCs were seeded in six-well plates and treated with osteogenesis induction medium, which was high-glucose Dulbecco's modified Eagle's medium containing 10\% FBS (Gibco, Raritan, NJ, USA), $10 \mathrm{mM}$ $\beta$-glycerophosphate (Sigma, St. Louis, MO, USA) and $0.2 \mathrm{mM}$ ascorbic acid (Sigma). 


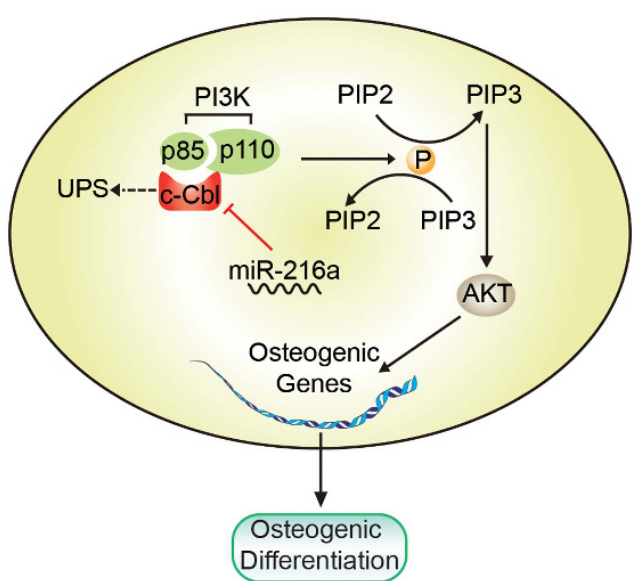

Figure 8 A schematic model for the miR-216a-mediated promotion of the osteogenic differentiation of hMSCs. miR-216a suppresses c-Cbl translation at the post-transcriptional level, resulting in the enhancement of the PI3K/AKT pathway and thereby promoting osteogenic differentiation

The medium was changed every 3 days. To verify the effect of DEX on osteogenic differentiation, different concentrations of $\operatorname{DEX}(1,10,100$ or $1000 \mathrm{nM})$ were added to the osteogenesis induction medium. To clarify the effects of the PI3K pathway on osteogenic differentiation, different concentrations $(5,10$ or $20 \mu \mathrm{M})$ of LY294002 (Selleck, Houston, TX, USA) were added to the osteogenesis induction medium. To investigate the effects of the STAT5 pathway on osteogenic differentiation, cells were treated with STAT5 inhibitor (Sc-355979; $100 \mu \mathrm{M})$ or solvent (dimethylsulfoxide; $0.1 \%$ ).

ALP and Alizarin red staining. ALP staining was monitored using an ALP staining kit (Institute of Hematology and Blood Diseases Hospital, Chinese Academy of Medical Sciences, Tianjin, China) according to the manufacturer's protocol. For Alizarin red staining (Sigma), cells were washed with PBS, fixed in $4 \%$ paraformaldehyde for 10 min, rinsed with double-distilled $\mathrm{H}_{2} \mathrm{O}$ and stained with $0.5 \%$ Alizarin red with $\mathrm{pH} 4.2$ for $30 \mathrm{~min}$ at room temperature. Cells were then rinsed with double-distilled $\mathrm{H}_{2} \mathrm{O}$ to remove the unbound dye and were subsequently photographed.

Assay for ALP activity. We applied the method that has been described previously. ${ }^{55}$ The cells were washed three times with PBS and lysed in $10 \mathrm{mM}$ Tris, $\mathrm{pH} 7.4,0.2 \%$ Igepal and $2 \mathrm{mM}$ phenylmethylsulfonyl fluoride. After sonication and centrifugation, the ALP activity in the supernatant was measured photometrically using the Alkaline Phosphatase Yellow Liquid Substrate System (pNPP, Sigma). Briefly, $5 \mu \mathrm{l}$ of thawed cell lysates were incubated with $200 \mu \mathrm{l}$ of the pNPP reagent for $30 \mathrm{~min}$ at $37^{\circ} \mathrm{C}$. The reaction was blocked by adding $50 \mu \mathrm{l}$ of $3 \mathrm{M} \mathrm{NaOH}$. The photometric values were determined using a spectrophotometer at $405 \mathrm{~nm}$. The ALP activity was normalized to the total protein of the cell lysates.

RNA extraction and qRT-PCR. Total RNA was extracted using TRIzol reagent (Invitrogen, Paisley, UK) and then was treated with DNase I (Ambion, Paisley, UK) at $37^{\circ} \mathrm{C}$ for $30 \mathrm{~min}$. CDNAs were synthesized using a high-capacity cDNA reverse transcription Kit (Applied Biosystems, Foster City, CA, USA). For the qRT-PCR analysis of miR-216a, the TaqMan miRNA reverse transcription kit and TaqMan miRNA assay kit (Applied Biosystems) were used. The relative expression of mRNA or miRNA was evaluated by the $2^{-\Delta \Delta C t}$ method and normalized to the expression of GAPDH or $\mathrm{U} 6$, respectively.

miRNA microarray analysis. miRNA expression profiling was performed using the TaqMan Array Human MicroRNA Cards V3.0 (Applied Biosystems, P/N: 4444913 ) following the manufacturer's instructions. The detailed information about the platform can be found on $\mathrm{ABI}$ website (https://products.appliedbiosystems.com). Experiments were performed twice using uninduced hAMSCs and in triplicate using osteogenic-induced 4-day cells. Ten nanograms of total RNA was used for each reaction. The criterion to determine the expression of miRNA is based on the CT value. miRNAs with CT value $>40$ in all compared groups were filtered. Only data with $P$-value $<0.05$ were included for further analyses. The expression of each individual miRNA was plotted as average CT values for each sample minus the average values for $U 6$ using the $2^{-\Delta \Delta C T}$ method. The data were adjusted by log transformation, mean centering, median centering and then normalization. Hierarchical clustering was manipulated by using average linkage method with Person correlation as a distance. Clustering of data and heat-map representations were performed by using Cluster 3.0 and Treeview software (http://smd.princeton.edu/resources/restech.shtml). TagMan qRT-PCR was used to validate the microarray data and the PCRs were performed at least in triplicate.

Western blot analysis. Cells were lysed in RIPA lysis buffer (Beyotime, Shanghai, China) with $1 \mathrm{mM}$ PMSF and protease inhibitor cocktail on ice for $30 \mathrm{~min}$, manually scraped from culture plates and then sonicated and quantified using the BCA Protein Assay Kit (Beyotime). Protein fractions were separated by $10 \%$ SDSPAGE and then were transferred to polyvinylidene difluoride membranes $(0.22 \mu \mathrm{m}$, Millipore, Billerica, MA, USA). The membranes were blocked with 5\% BSA and incubated with specific antibodies overnight at $4^{\circ} \mathrm{C}$ and then were incubated with horseradish peroxidase-conjugated secondary antibody for $1 \mathrm{~h}$ at room temperature. Immunodetection was visualized using a chemiluminescent ECL reagent (Millipore).

Ectopic bone formation in vivo. A total of $2 \times 10^{6}$ hAMSCs were mixed with wetted HA/TCP ceramic powder (80 mg; National Engineering Research Center for Biomaterials, Chengdu, China), incubated at $37^{\circ} \mathrm{C}$ overnight and implanted subcutaneously into the dorsal surface of 8 -week-old NOD/SCID mice. ${ }^{56}$ The implants were harvested at 8 weeks, fixed in $4 \%$ paraformaldehyde, decalcified in 10\% EDTA and embedded in paraffin. Thin sections $(5 \mu \mathrm{m})$ were stained with H\&E.

Clinical bone specimen preparation. Sixty-seven clinical bone specimens were collected from the Orthopaedic Beijing Union Medical College Hospital. Patients who had a fracture caused by falling without obvious violence were included in our study (inclusive criteria). Individuals with diabetes, malignancy or other severe diseases in the previous 5 years were excluded from our study (exclusive criteria). All of the clinical procedures were approved by the ethics committee at the Chinese Academy of Medical Sciences and Peking Union Medical College.

Dual luciferase reporter system luciferase assay. An 80- to 100-bp synthetic fragment of the 3UTR of c-Cbl, Smad7, NLK, CEBPG or TGFBR2 containing the putative miR-216a recognition element and corresponding mutated 3UTR were subcloned into the psiCHECK-2 vector (Promega, Southampton, UK) downstream of the renilla luciferase reporter gene. Transient co-transfection of HEK293T cells $\left(1 \times 10^{5}\right.$ cells per well) with the luciferase reporter vector and miR-216a mimic or miR-negative control (miR-NC) was performed in 24-well plates using Lipofectamine 2000 (Invitrogen). The luciferase activities were measured $48 \mathrm{~h}$ after transfection using the Dual Luciferase Reporter Assay System (Promega), and each experiment was repeated in triplicate. The renilla luciferase activity was normalized to the firefly luciferase activity for each transfected well.

Statistical analysis. All of the numerical results are presented as the means \pm S.D. Significant differences between groups were analyzed using Student's $t$-tests. For multiple-group testing, analysis of variance was conducted followed by a posteriori $t$-testing. Differences were considered statistically significant at ${ }^{\star} P<0.05,{ }^{\star *} P<0.01$ and ${ }^{\star \star *} P<0.001$.

\section{Conflict of Interest}

The authors declare no conflict of interest.

Acknowledgements. This work was supported by grants from the National Natural Science Foundation of China (no. 81370466 and no. 81272009), the National Key Scientific Program of China (no. 2011CB964901), National Collaborative Innovation Program, and Program for Cheung Kong Scholars and Innovative Research Team in University-PCSIRT (no. IRT0909), and the National Science and Technology Major Projects for 'Drug Research and Development' (2014ZX09101042).

1. Ahmed SF, Elmantaser M. Secondary osteoporosis. Endocr Dev 2009; 16: 170-190.

2. Canalis E, Delany AM. Mechanisms of glucocorticoid action in bone. Ann N Y Acad Sci 2002; 966: 73-81.

3. Yun SI, Yoon HY, Jeong SY, Chung YS. Glucocorticoid induces apoptosis of osteoblast cells through the activation of glycogen synthase kinase 3beta. J Bone Miner Metab 2009; 27: 140-148. 
4. Pittenger MF, Mackay AM, Beck SC, Jaiswal RK, Douglas R, Mosca JD et al. Multilineage potential of adult human mesenchymal stem cells. Science 1999; 284: 143-147.

5. Zuk PA, Zhu M, Ashjian P, De Ugarte DA, Huang Jl, Mizuno H et al. Human adipose tissue is a source of multipotent stem cells. Mol Biol Cell 2002; 13: 4279-4295.

6. Mosna F, Sensebe L, Krampera M. Human bone marrow and adipose tissue mesenchymal stem cells: a user's guide. Stem Cells Dev 2010; 19: 1449-1470.

7. Hass R, Kasper C, Bohm S, Jacobs R. Different populations and sources of human mesenchymal stem cells (MSC): a comparison of adult and neonatal tissue-derived MSC. Cell Commun Signal 2011; 9: 12

8. Chamberlain G, Fox J, Ashton B, Middleton J. Concise review: mesenchymal stem cells their phenotype, differentiation capacity, immunological features, and potential for homing Stem Cells 2007; 25: 2739-2749.

9. Fink T, Rasmussen JG, Emmersen J, Pilgaard L, Fahlman A, Brunberg $S$ et al. Adipose derived stem cells from the brown bear (Ursus arctos) spontaneously undergo chondrogenic and osteogenic differentiation in vitro. Stem Cell Res 2011; 7: 89-95.

10. Lips $P$, Courpron P, Meunier PJ. Mean wall thickness of trabecular bone packets in the human iliac crest: changes with age. Calcif Tissue Res 1978; 26: 13-17.

11. Kusumbe AP, Ramasamy SK, Adams RH. Coupling of angiogenesis and osteogenesis by a specific vessel subtype in bone. Nature 2014; 507: 323-328.

12. Yang M, Liu R, Li X, Liao J, Pu Y, Pan E et al. miRNA-183 suppresses apoptosis and promotes proliferation in esophageal cancer by targeting PDCD4. Mol Cells 2014; 37: 873-880.

13. Mori M, Triboulet R, Mohseni M, Schlegelmilch K, Shrestha K, Camargo FD et al. Hippo signaling regulates microprocessor and links cell-density-dependent miRNA biogenesis to cancer. Cell 2014; 156: 893-906.

14. Ventura A, Young AG, Winslow MM, Lintault L, Meissner A, Erkeland SJ et al. Targeted deletion reveals essential and overlapping functions of the miR-17 through 92 family of miRNA clusters. Cell 2008; 132: 875-886.

15. Brennecke J, Hipfner DR, Stark A, Russell RB, Cohen SM. Bantam encodes a developmentally regulated microRNA that controls cell proliferation and regulates the proapoptotic gene hid in Drosophila. Cell 2003; 113: 25-36.

16. Poy MN, Eliasson L, Krutzfeldt J, Kuwajima S, Ma X, Macdonald PE et al. A pancreatic isletspecific microRNA regulates insulin secretion. Nature 2004; 432: 226-230.

17. Carthew RW, Sontheimer EJ. Origins and mechanisms of miRNAs and siRNAs. Cell 2009; 136: $642-655$

18. Huntzinger $\mathrm{E}$, Izaurralde $\mathrm{E}$. Gene silencing by microRNAs: contributions of translational repression and mRNA decay. Nat Rev Genet 2011; 12: 99-110.

19. He L, Hannon GJ. MicroRNAs: small RNAs with a big role in gene regulation. Nat Rev Genet 2004; 5: 522-531.

20. Zamore PD, Haley B. Ribo-gnome: the big world of small RNAs. Science 2005; $\mathbf{3 0 9}$ $1519-1524$.

21. Lian JB, Stein GS, van Wijnen AJ, Stein JL, Hassan MQ, Gaur T et al. MicroRNA control of bone formation and homeostasis. Nat Rev Endocrinol 2012; 8: 212-227.

22. Li Z, Hassan MQ, Volinia S, van Wijnen AJ, Stein JL, Croce CM et al. A microRNA signature for a BMP2-induced osteoblast lineage commitment program. Proc Natl Acad Sci USA 2008; 105: 13906-13911.

23. Eskildsen T, Taipaleenmaki H, Stenvang J, Abdallah BM, Ditzel N, Nossent AY et al. MicroRNA-138 regulates osteogenic differentiation of human stromal (mesenchymal) stem cells in vivo. Proc Natl Acad Sci USA 2011; 108: 6139-6144.

24. Wei J, Shi Y, Zheng L, Zhou B, Inose H, Wang J et al. miR-34s inhibit osteoblast proliferation and differentiation in the mouse by targeting SATB2. J Cell Biol 2012; 197: 509-521.

25. Yu S, Geng Q, Ma J, Sun F, Yu Y, Pan Q et al. Heparin-binding EGF-like growth factor and miR-1192 exert opposite effect on Runx2-induced osteogenic differentiation. Cell Death Dis 2013; 4: e868.

26. Wang X, Guo B, Li Q, Peng J, Yang Z, Wang A et al. miR-214 targets ATF4 to inhibit bone formation. Nat Med 2013; 19: 93-100.

27. Chen L, Holmstrom K, Qiu W, Ditzel N, Shi K, Hokland L et al. MicroRNA-34a inhibits osteoblast differentiation and in vivo bone formation of human stromal stem cells. Stem Cells 2014; 32: 902-912

28. Kureel J, Dixit M, Tyagi AM, Mansoori MN, Srivastava K, Raghuvanshi A et al. miR-542-3p suppresses osteoblast cell proliferation and differentiation, targets BMP-7 signaling and inhibits bone formation. Cell Death Dis 2014; 5: e1050.

29. Zuo B, Zhu JF, Li J, Wang CD, Zhao XY, Cai GQ et al. MicroRNA-103a functions as a mechanosensitive microRNA to inhibit bone formation through targeting Runx2. J Bone Miner Res 2015; 30: 330-345

30. Huang J, Zhao L, Xing L, Chen D. MicroRNA-204 regulates Runx2 protein expression and mesenchymal progenitor cell differentiation. Stem Cells 2010; 28: 357-364.

31. Li HL, Li TP, Wang SH, Wei JF, Fan JF, Li J et al. miR-17-5p and miR-106a are involved in the balance between osteogenic and adipogenic differentiation of adipose-derived mesenchymal stem cells. Stem Cell Res 2013; 10: 313-324.
32. Zhang JF, Fu WM, He ML, Wang H, Wang WM, Yu SC et al. MiR-637 maintains the balance between adipocytes and osteoblasts by directly targeting Osterix. Mol Biol Cell 2011; 22: 3955-3961.

33. Wang FS, Chuang PC, Lin CL, Chen MW, Ke HJ, Chang YH et al. MicroRNA-29a protects against glucocorticoid-induced bone loss and fragility in rats by orchestrating bone acquisition and resorption. Arthritis Rheum 2013; 65: 1530-1540.

34. Ko JY, Chuang PC, Chen MW, Ke HC, Wu SL, Chang YH et al. MicroRNA-29a ameliorates glucocorticoid-induced suppression of osteoblast differentiation by regulating beta-catenin acetylation. Bone 2013; 57: 468-475.

35. Overman RA, Toliver JC, Yeh JY, Gourlay ML, Deal CL. U.S. adults meeting 2010 American College of Rheumatology criteria for treatment and prevention of glucocorticoid-induced osteoporosis. Arthritis Care Res (Hoboken) 2014; 66: 1644-1652.

36. Li J, Zhang N, Huang X, Xu J, Fernandes JC, Dai K et al. Dexamethasone shifts bone marrow stromal cells from osteoblasts to adipocytes by C/EBPalpha promoter methylation. Cell Death Dis 2013; 4: e832.

37. Thien CB, Langdon WY. Cbl: many adaptations to regulate protein tyrosine kinases. Nat Rev Mol Cell Biol 2001; 2: 294-307.

38. Schmidt MH, Dikic I. The Cbl interactome and its functions. Nat Rev Mol Cell Biol 2005; 6 : 907-918.

39. Brennan T, Adapala NS, Barbe MF, Yingling V, Sanjay A. Abrogation of Cbl-PI3K interaction increases bone formation and osteoblast proliferation. Calcif Tissue Int 2011; 89: 396-410.

40. Adapala NS, Holland D, Scanlon V, Barbe MF, Langdon WY, Tsygankov AY et al. Loss of $\mathrm{Cbl}-\mathrm{PI} 3 \mathrm{~K}$ interaction in mice prevents significant bone loss following ovariectomy. Bone 2014; 67: 1-9

41. Dieudonne FX, Severe N, Biosse-Duplan M, Weng JJ, Su Y, Marie PJ. Promotion of osteoblast differentiation in mesenchymal cells through Cbl-mediated control of STAT5 activity. Stem Cells 2013; 31: 1340-1349.

42. Kang $\mathrm{IH}$, Jeong $\mathrm{BC}$, Hur SW, Choi $\mathrm{H}$, Choi SH, Ryu JH et al. MicroRNA-302a stimulates osteoblastic differentiation by repressing COUP-TFll expression. J Cell Physiol 2015; 230: 911-921.

43. Cheung KS, Sposito N, Stumpf PS, Wilson DI, Sanchez-Elsner T, Oreffo RO. MicroRNA-146a regulates human foetal femur derived skeletal stem cell differentiation by down-regulating SMAD2 and SMAD3. PLoS One 2014; 9: e98063.

44. Shapiro F. Bone development and its relation to fracture repair. The role of mesenchymal osteoblasts and surface osteoblasts. Eur Cell Mater 2008; 15: 53-76.

45. Park D, Spencer JA, Koh BI, Kobayashi T, Fujisaki J, Clemens TL et al. Endogenous bone marrow MSCs are dynamic, fate-restricted participants in bone maintenance and regeneration. Cell Stem Cell 2012; 10: 259-272.

46. OBrien CA, Jia D, Plotkin LI, Bellido T, Powers CC. Stewart SA et al. Glucocorticoids act directly on osteoblasts and osteocytes to induce their apoptosis and reduce bone formation and strength. Endocrinology 2004; 145: 1835-1841.

47. Grigoriadis AE, Heersche JN, Aubin JE. Differentiation of muscle, fat, cartilage, and bone from progenitor cells present in a bone-derived clonal cell population: effect of dexamethasone. J Cell Biol 1988; 106: 2139-2151.

48. Kato M, Putta S, Wang M, Yuan H, Lanting L, Nair I et al. TGF-beta activates Akt kinase through a microRNA-dependent amplifying circuit targeting PTEN. Nat Cell Biol 2009; 11: 881-889.

49. Zhang J, Ning $X$, Cui W, Bi M, Zhang D. Transforming growth factor (TGF)-beta-induced microRNA-216a promotes acute pancreatitis via Akt and TGF-beta pathway in mice. Dig Dis Sci 2015; 60: 127-135.

50. Ueno H, Sasaki K, Honda H, Nakamoto T, Yamagata T, Miyagawa K et al. c-Cbl is tyrosinephosphorylated by interleukin-4 and enhances mitogenic and survival signals of interleukin-4 receptor by linking with the phosphatidylinositol 3 '-kinase pathway. Blood 1998; 91: 46-53.

51. Adapala NS, Barbe MF, Langdon WY, Nakamura MC, Tsygankov AY, Sanjay A. The loss of Cbl-phosphatidylinositol 3-kinase interaction perturbs RANKL-mediated signaling, inhibiting bone resorption and promoting osteoclast survival. J Biol Chem 2010; 285: 36745-36758.

52. Adapala NS, Barbe MF, Tsygankov AY, Lorenzo JA, Sanjay A. Loss of Cbl-PI3K interaction enhances osteoclast survival due to p21-Ras mediated PI3K activation independent of Cbl-b. J Cell Biochem 2014; 115: 1277-1289.

53. Huang S, Wang S, Bian C, Yang Z, Zhou H, Zeng Y et al. Upregulation of miR-22 promotes osteogenic differentiation and inhibits adipogenic differentiation of human adipose tissuederived mesenchymal stem cells by repressing HDAC6 protein expression. Stem Cells Dev 2012; 21: 2531-2540

54. Can A, Balci D. Isolation, culture, and characterization of human umbilical cord stromaderived mesenchymal stem cells. Methods Mol Biol 2011; 698: 51-62.

55. Addison WN, Azari F, Sorensen ES, Kaartinen MT, McKee MD. Pyrophosphate inhibits mineralization of osteoblast cultures by binding to mineral, up-regulating osteopontin, and inhibiting alkaline phosphatase activity. J Biol Chem 2007; 282: 15872-15883.

56. Abdallah BM, Ditzel N, Kassem M. Assessment of bone formation capacity using in vivo transplantation assays: procedure and tissue analysis. Methods Mol Biol 2008; 455: 89-100. 